\title{
Evidence Implicating Descending Fibers in Self-Stimulation of the Medial Forebrain Bundle
}

\author{
Catherine Bielajew ${ }^{1}$ and Peter Shizgal \\ Center for Studies in Behavioral Neurobiology, Department of Psychology, Concordia University, \\ Montreal, Quebec, H3G 1M8, Canada
}

The role of ascending and descending fibers in self-stimulation of the lateral hypothalamus and ventral tegmental area in the rat was assessed by noting whether anodal hyperpolarization of one of these sites could reduce the rewarding effect of stimulating the other site.

Strength-duration curves were obtained by psychophysical means, with one of the depth electrodes serving as the cathode and the other as the anode. It was anticipated that at long pulse durations, conduction in some of the fibers stimulated at the cathode would be blocked at the anode. At shorter durations, the anodal hyperpolarization should have dissipated before the arrival of the action potentials triggered by the cathode. Thus, the predicted effect of the block was to bend the strength-duration curves obtained with two depth electrodes upward at long pulse durations, provided that the anode lay between the cathode and the efferent stages of the pathway responsible for the rewarding effect.

To control for possible differences in the density of the reward substrate in the lateral hypothalamic and ventral tegmental areas, the strength-duration curves obtained with a given cathode and a depth anode were compared to curves obtained with the same cathode but with an anode consisting of a set of skull screws. It was expected that the concentrated current entering from the depth anode would much more effectively block conduction in the medial forebrain bundle than the diffuse current entering from the large, distant skull screws.

The predicted change in the shape of the strength-duration curves was observed only when the ventral tegmental electrode served as the anode and the lateral hypothalamic electrode as the cathode. This is consistent with the notion that in at least some of the neurons responsible for the rewarding effect, action potentials elicited by the lateral hypothalamic electrode had to pass through the ventral tegmental area in order to reach the efferent stages of the reward pathway. In the simplest anatomical arrangement consonant with this view, the somata of these cells lie in the forebrain and give rise to descending axons.

As a test of the hypothesis that anodal block was responsible for changing the shape of the strength-duration curve obtained with the ventral tegmental anode, a psychophysical version of

\footnotetext{
Received July 12, 1984; revised Oct. 4, 1985; accepted Oct. 10, 1985.

This work was supported by NSERC of Canada Grant A0308 and Grant EQ09 from the Fonds F.C.A.C. du Quebec. We wish to thank George Fouriezos and the School of Psychology at the University of Ottawa for providing space in which to conduct a portion of this study. Wc also thank Ivan Kiss, Sylvie Laniel, and William Mundl for their technical assistance and C. R. Gallistel, Leslie Paris, Roy Wise, and John Yeomans for their helpful comments on the manuscript.

Correspondence should be addressed to P. Shizgal, Center for Studies on Behavioral Neurobiology, Department of Psychology, Concordia University, 1455 de Maisonneuve Blvd. West, Rm. H-1013-4, Montreal, Quebec, H3G 1M8, Canada.

' Present address: School of Psychology, University of Ottawa, Ottawa, Ontario K1N 6N5, Canada.

Copyright $\odot 1986$ Society for Neuroscience $0270-6474 / 86 / 040919-11 \$ 02.00 / 0$
}

the collision test was used to determine whether the tips of the lateral hypothalamic and ventral tegmental electrodes were indeed linked by a common set of reward-related fibers. The results of this test were positive when the sizes of the stimulation fields were adjusted to be comparable to those used in the direction test. In the final test, the field size was reduced, lowering both the probability that the same fibers would be fired by the two electrodes in the collision test and the probability that an action potential triggered by the cathode would be blocked at the anode in the direction test. As predicted, this manipulation eliminated both the collision effect and the change in the shape of the strength-duration curves obtained with the ventral tegmental anode.

Among the most striking effects of electrically stimulating the medial forebrain bundle (MFB) is the pronounced enduring potentiation of subsequent behavior directed at obtaining additional stimulation (self-stimulation). Evidence linking this rewarding effect to those of natural stimuli such as food (Hoebel, 1974; Rolls et al., 1980) provides encouragement that unraveling the circuitry subserving self-stimulation of the MFB may shed light on the neural substrates for naturally occurring appetitive behaviors. It has also been argued (Fibiger, 1978; Wise, 1978,1980 ) that dependence-inducing drugs attain their powerful grip on behavior through their action on the neural substrate for MFB reward. Hence, it is not surprising that interest in brain stimulation reward has endured throughout the long, but as yet unsuccessful, search for its substrate.

Why has the identification of these neurons been so difficult? In part the problem lies with the anatomical complexity of the regions from which the most vigorous self-stimulation has been elicited. For example, the MFB appears to include about 50 different components that can be distinguished on the basis of origin and destination (Nieuwenhuys et al., 1982). In order to identify the neurons responsible for brain stimulation reward, one must have some means of distinguishing these cells from other neurons fircd by the electrode.

The psychophysical approach to the study of brain stimulation reward (Deutsch, 1964; Gallistel, 1975; Gallistel et al., 1981) was developed in order to deal with this problem. The goal of the approach is to infer anatomical and physiological properties of the substrate from the results of appropriately designed behavioral experiments. Because of the manner in which they are derived, these characteristics are believed to pertain only to the neurons responsible for the rewarding effect and not to other stimulated cells. If so, they provide criteria for evaluating the likelihood that a given set of electrophysiologically or anatomically identified neurons constitutes part of the substrate for self-stimulation.

On the basis of psychophysical work carried out over the past decade, an increasingly detailed portrait is emerging of the neurons responsible for self-stimulation of the MFB. These studies 


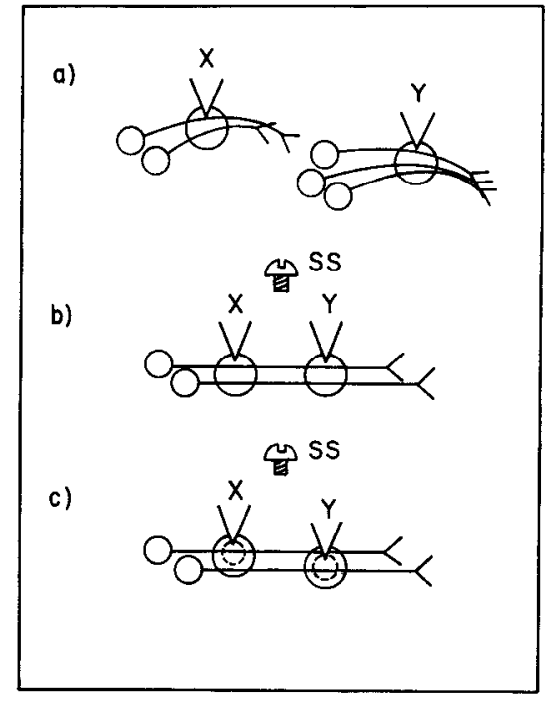

Figure 1. Illustration of the rationale for the direction and collision tests. The difference between $a$ and $b$ explains why the key comparisons in the direction test were carried out using a given depth clectrode as the cathode and either the second depth electrode or the skull screw $(S S)$ as the anode. If only the depth electrodes were used and the effects of passing current from electrode $X$ to electrode $Y$ compared to those obtained with the direction of current flow reversed, then the same behavioral outcome could arise from very different anatomical relationships between the reward fibers coursing past the tips of the two depth electrodes. In both $a$ and $b$, the stimulation would be least effective when $X$ served as the cathode and $Y$ as the anode. However, in $a$ this would be due to the difference in the density of the reward substrate at the two sites, whereas in $b$ this would be due to anodal block. The effect of varying the current in the collision test is illustrated in $c$. The solid circles represent the effective stimulation fields associated with high currents. A common set of neurons passes through both fields, and a collision effect is obtaincd. When the current is reduced to yield the smaller fields denoted by the dotted circles, no neurons pass through both fields, and the collision effect is eliminated. If the region in which anodal block occurs is no larger than the region in which a current of the same magnitude fires neurons, then the reduction in current that eliminates the collision effect will also eliminate the behavioral effect of the anodal block: The neurons in which the block occurs at the lower current will no longer be stimulated at the cathode.

suggest that fine, myelinated axons with conduction velocities of 1-8 m/sec (Bielajew and Shizgal, 1982; Shizgal et al., 1980a) and refractory periods of $0.5-1.2 \mathrm{msec}$ (Yeomans, 1975, 1979) make up at least part of the directly stimulated substrate. The present experiment was undertaken to add a crucial feature to this characterization by inferring the direction in which these neurons project.

For stylistic reasons, we will refer to the neurons responsible for the rewarding effect of brain stimulation as "reward neurons" and to the information they carry as "reward signals." This nomenclature is intended to imply neither that these neurons are devoted exclusively to a single function nor that they constitute a final common path through which the effects of all rewarding stimuli are funneled.

The root idea of the technique used here (Shizgal et al., 1980b) is to employ an anodal hyperpolarization block to temporarily interfere with conduction between a self-stimulation electrode and the terminals that transmit the reward signals to the next stage of the substrate. Direction is inferred by noting which of a pair of MFB self-stimulation electrodes produces a behaviorally effective block. The ineffective electrode is presumed to block antidromic conduction between the second electrode and the somata, leaving unaltered the distal part of the axon.
Figure 1 illustrates the need for adding a third electrode in order to distinguish between an anodal block and another mechanism that could account for a difference in the effectiveness of stimulation when the direction of current flow is reversed. Different fibers pass by the two electrode tips in Figure $1 a$, while the same fibers pass by the two tips in Figure $1 b$. In both cases, the effect of stimulating with depth electrode $\mathrm{X}$ as the cathode and depth electrode $Y$ as the anode should be weaker than the effect of stimulating with $\mathrm{Y}$ as the cathode and $\mathrm{X}$ as the anode. However, in Figure $1 b$ this difference is due to an anodal hyperpolarization block, while in Figure $1 a$ it is due to the different densities of reward neurons at the two electrode tips and the fact that excitation occurs principally at the cathode (Ranck, 1981).

The use of a set of skull screws as an alternative anode eliminates this ambiguity. When the depth electrode serves as the anode, the hyperpolarizing current is concentrated at the tip and may block the conduction of orthodromic impulses generated at the cathode. In contrast, when the anode is the set of large remote screws, the hyperpolarizing current should be too diffuse to interfere with conduction. Note that the density of reward neurons at the stimulation site is held constant because the same electrode serves at the cathode. Thus, one test of the hypothesis that anodal hyperpolarization at a given level of the MFB blocks the conduction of reward signals elicited at another level of the MFB is to determine whether the effectiveness of the stimulation is restored by substituting a skull screw anode for the depth anode.

When monophasic current pulses are passed through the two depth electrodes in series, propagation of the impulses initiated at the cathode can be blocked by hyperpolarization at the anode only if the pulse duration approaches or exceeds the interelectrode conduction time (Kuffler and Vaughan-Williams, 1953). At shorter durations the hyperpolarization will have dissipated before the action potentials arrive. This relationship provides a second means of testing the hypothesis that anodal hyperpolarization at a given MFB site blocks the conduction of reward signals. Thus, we set up the direction test as a behavioral strengthduration experiment (Matthews, 1977).

Assume that with the arrangement depicted in Figure $1 b$, a strength-duration curve is obtained using electrode $X$, the proximal electrode, as the cathode, and the skull screws as the anode. This curve can then be compared to one obtained using $X$ as the cathode and electrode $Y$, the distal electrode, as the anode. Hyperpolarization of the region under $Y$ may block the conduction of impulses triggered by $X$, but only when the pulse duration is sufficiently long. Hence, at the longer pulse durations, the strength-duration curve obtained with $Y$ as the anode should deviate from the curve obtained with the skull screw anode: More current should be required in order to compensate for the loss of the impulses blocked at the anode.

Different results are expected when $Y$ serves as the cathode. Since the anodal block is now interposed between the soma and the cathode, it is not expected to alter the rewarding effect; which depends on the synaptic output of the stimulated cells. Furthermore, since the shape of a strength-duration curve is expected to be independent of the angle of current flow with respect to the fibers (Rushton, 1927), both strength-duration curves obtained with electrode $\mathrm{Y}$ as the cathode should be similar in shape.

Figure 1, $b$ and $c$, illustrates two additional means of testing the hypothesis that reward signals are blocked by anodal hyperpolarization. A psychophysical technique based on the phenomenon of collision (Shizgal et al., 1980a) provides an independent means of determining whether the two self-stimulation sites are indeed linked by the axons of MFB reward neurons. Effectively, this test asks whether Figure $1, a$ or $b$, better de- 
scribes the trajectories of the directly stimulated reward neurons with respect to the two depth electrodes. With this method, pairs of stimulation pulses are delivered using two cathodes aimed at self-stimulation sites along a given fiber bundle and a skull screw anode. The first pulse in each pair (C) is applied through one cathode and the second pulse $(T)$ through the other. In fibers stimulated by both electrodes, collision between orthodromic and antidromic action potentials will occur when the C-T interval is less than the sum of the interelectrode conduction time and the axonal refractory period. If reward fibers are among the population stimulated by both electrodes, then the effectiveness of the stimulation in producing a rewarding effect will vary with the $C-T$ interval. At short intervals, it will be necessary to increase the strength of the stimulation in order to compensate for the collision block. Thus, the third criterion for attributing an effect to anodal hyperpolarization block is that a collision-like effect be demonstrated using the same electrodes as in the direction test and cathodal stimulation fields of comparable size (depicted by the solid circles in Fig. 1c).

The dotted lines in Figure $1 c$ represent the smaller regions that would be excited by weaker currents flowing from the skull screws to either of the depth electrodes. In this case, no reward neurons pass through both fields, and the collision test will yield negative results. Given that the cathode and anode are in series in the direction test, a reduction in the size of the cathodal field brings about a corresponding reduction in the size of the region in which anodal block occurs. If the region stimulated by a given anodal current is no larger than the region stimulated by a cathodal current of the same magnitude and duration (see below), then impulses triggered by a cathodal field of the size depicted by the dotted lines in Figure $1 c$ should escape anodal block. If so, strength-duration curves obtained using these smaller fields should be similar in shape regardless of whether a depth electrode or the skull screw serves as the anode. Thus, manipulation of the size of the effective fields provides a fourth test of the hypothesis that anodal hyperpolarization at one level of the MFB blocks the conduction of reward signals triggered at another level of the MFB: The difference in the shape of the strength-duration functions should disappear when the field size is reduced to a level that fails to yield collision.

\section{Materials and Methods}

\section{Surgery}

Male hooded rats of the Long-Evans strain (Canadian Breeding Farms and Laboratories) weighing from $300-450 \mathrm{gm}$ were separately housed in wire-mesh or plastic cages and maintained on a $12 \mathrm{hr}$ light $/ 12 \mathrm{hr}$ dark cycle. Food and water were available freely. Prior to surgery, the animals were anesthetized with sodium pentobarbital $(60 \mathrm{mg} / \mathrm{kg}$, i.p.). Using standard stereotaxic procedures, electrodes were aimed at the left lateral hypothalamus (LH) and ventral tegmental area (VTA). With the incisor bar set $5.0 \mathrm{~mm}$ above the interaural line, the following coordinates were used: $\mathrm{LH}-0.4 \mathrm{~mm}$ behind bregma, $1.7 \mathrm{~mm}$ lateral to the sagittal suture, and 7.5-8.0 mm below the dura meter; VTA-3.3 mm behind bregma, $0.7 \mathrm{~mm}$ lateral to the sagittal suture, and $7.5-8.0 \mathrm{~mm}$ below the dura mater.

Electrodes were fashioned from $0.25 \mathrm{~mm}$ stainless steel wire insulated with Formvar to within roughly $0.25 \mathrm{~mm}$ of the rounded tip. A fine stainless steel wire wrapped around four stainless steel anchoring screws served as the anode unless stated otherwise. The screws straddled the sagittal suture; two were located anterior to bregma and two were located near lambda. The entire assembly was secured to the skull with dental cement.

\section{Apparatus}

The test chamber ( $25 \mathrm{~cm}$ wide, $25 \mathrm{~cm}$ deep, $70 \mathrm{~cm}$ high) was constructed of Plexiglas and was fitted with a grid floor. A Lehigh Valley rodent lever protruded into the lower-right corner of the left wall about $6 \mathrm{~cm}$ above the floor.
Stimulation consisted of $0.5 \mathrm{sec}$ trains of monopolar rectangular cathodal pulses. The temporal parameters of the stimulation were controlled by integrated-circuit pulse generators and the amplitude by dual constant-current amplifiers (Mundl, 1980). In the absence of input pulses, the outputs of each channel of the constant-current amplifier were shorted through $1 \mathrm{k} \Omega$ resistors to prevent the build-up of charge at the electrode-brain interface.

\section{Testing procedures}

\section{Screening and stabilization}

After a postoperative recovery period of $2-3 \mathrm{~d}$, each subject was screcncd for self-stimulation; the method of successive approximations was used to shape the animal to the lever. In order to pass into the next phase of the experiment, a subject had to lever-press vigorously ( $>30$ responses/min for optimal parameters) for stimulation of the $\mathrm{LH}$ and of the VTA.

Performance of the self-stimulation task was stabilized using the same procedure for both sites. Starting with the parameters found to support high rates of responding, the current was held constant and the number of pulses was reduced by $0.1 \log _{10}$ steps after every trial until response rates near zero were observed. A $15 \mathrm{sec}$ pause separated the $30 \mathrm{sec}$ trials. This procedure was repeated until the number of pulses required to sustain a half-maximal rate of lever pressing (hereafter referred to as the "required number") did not vary by more than $0.1 \log _{10}$ units across five determinations. Gencrally three sessions were required to attain the stability criterion.

\section{Pilot collision tests}

The purpose of these pilot tests was to assess quickly whether the two stimulation fields were sufficiently large and well aligned to activate a common set of reward fibers. These tests served to choose optimal current values for subsequent tests and to screen out subjects in which the electrodes appeared to be so misaligned as to preclude stimulating a common set of reward fibers at practical current levels.

The LH and VTA currents were adjusted to equate the required numbers of single pulses when each electrode was tested separately. Stimulation consisted of trains of pulse pairs, with the first member of each pair, the $C$ pulse, applied to one electrode and the second member of each pair, the $T$ pulse, applied to the other electrode. The required number of pulse pairs was determined for two $\mathrm{C}-\mathrm{T}$ intervals, 0.5 and 5 msec.

We reasoned that more pulse pairs would be required at short than at long C-T intervals in order to compensate for firings lost due to collision. If so, a collision effect should have been manifested in a difference in the required-number values for appropriately selected short and long C-T intervals. We chose C-T intervals of 0.5 and $5 \mathrm{msec}$ because they bracket the intervals at which we had previously found an abrupt increase in the required number of pulse pairs when stimulating similar MFB sites (Bielajew and Shizgal, 1982; Shizgal et al., 1980a).

If, after 10 determinations, there was no clear difference in the required number of pulse pairs at the short and long C-T intervals, the current at each site was raised, performance was restabilized, and the pilot test was rerun. This procedure was repeated until either a collisionlike effect was observed or the maximum current generated by the stimulators was reached (roughly $1.6 \mathrm{~mA}$ ).

\section{Collision and refractory period tests}

If the results of the pilot test were positive, full collision and refractory period tests were conducted. (Full tests were also conducted on one subject, SL-19, that did not pass the pilot test). In the collision test, both anterior-posterior (AP) and posterior-anterior (PA) conditions were tested. During the AP test, the C pulses were delivered to the $\mathrm{LH}$ and the $T$ pulses were delivered to the VTA; this arrangement was reversed in the PA condition. In the refractory period tests, the $\mathrm{C}$ and T pulses were both delivered to either the LH or the VTA; one electrode was always inactive. A typical test session comprised approximately 16 determinations of the required number: 12 determinations of the required number of pulse pairs and 4 determinations of the required number of single pulses. The latter were spaced at roughly equal intervals throughout the test session. In the collision test the determination of the required number of single pulses was carried out separately for each clectrode.

To summarize, a single data set consisted of two refractory period 
tests, one using the LH electrode and the other the VTA electrode and two collision tests, one with the C pulses delivered to the $\mathrm{LH}$ and the $T$ pulses delivered to the VTA and the other with the $C$ pulses delivered to the VTA and the T pulses delivered to the LH. Two to eight data sets were obtained with each subject.

\section{Data format}

Yeomans' scaling procedure (1975) was used to assess the effects of varying the $\mathrm{C}-\mathrm{T}$ interval in the refractory period tests. The relative effectiveness of the $T$ pulses was estimated using the following formula:

$$
E=R N_{\mathrm{SP}} / R N_{\mathrm{C}-\mathrm{T}}-1
$$

where $E$ is the effectiveness of double-pulse stimulation; $R N_{\mathrm{SP}}$, the required number of single pulses; $R N_{\mathrm{C}-\mathrm{T}}$, the required number of pulse pairs at a given $\mathrm{C}-\mathrm{T}$ interval. When $E=0, R N_{\mathrm{SP}}$ and $R N_{\mathrm{C}-\mathrm{T}}$ arc equal in value, indicating that the addition of the $T$ pulse has no behavioral effect. When $E=1$, the value of $R N_{\mathrm{SP}}$ is twice that of $R N_{\mathrm{C}-\mathrm{T}}$, indicating that the behavioral effect of the $T$ pulse is equal to that of the $C$ pulse.

The formula used to compute the effectiveness of double-pulse stimulation in the collision tests (Bielajew and Shizgal, 1982) was as follows:

$$
\left.E=\left[\left(R N_{\mathrm{SPL}} / R N_{\mathrm{C}-\mathrm{T}}\right)-1\right)\right] /\left(R N_{\mathrm{SPL}} / R N_{\mathrm{SPH}}\right)
$$

where $R N_{\mathrm{SPL}}$ is the required number of single pulses for the site with the lower required-number value and $R N_{\mathrm{SPH}}$ is the required number of single pulses for the site with the higher required-number value. As explained previously (Bielajew and Shizgal, 1982), this formula corrects for differences in the required number of single pulses at each placement.

\section{Direction test}

Two pairs of electrode configurations were tested in this final and crucial phase of the experiment. In the experimental conditions either depth electrode served as the cathode and the second depth electrode as the anode; it was in these conditions that an anodal block was anticipated. In the conditions used to control for variations in the density of reward neurons in the LH and VTA, either depth electrode served as the cathode and the skull screws as the anode. The key comparisons were made between the experimental and control conditions in which a given depth electrode served as the cathode. Note that the electrode configurations in which the skull screws served as the anode were identical to those used in the collision and refractory period tests.

The direction tests began after the collision and refractory period tests were completed. For each stimulation condition, the following procedure was employed: With the number of pulses held constant, the current was varied from a level yielding maximum performance to a level at which there was little or no responding. The required current, defined as the current that supported a half-maximal response rate, was computed by interpolation. The required current was determined for a series of pulse durations ranging from 0.1 to $6.4 \mathrm{msec}$ across subjects. Each subject underwent two to eight tests of each set of four electrode configurations.

The choice of the number of pulses to be used in the direction test is important because it determines the degree to which the results of the direction test are comparable to those of the collision and refractory period tests. The results are most meaningfully compared when all three tests are carried out on the same population of reward neurons. The region stimulated by a given cathode in the collision and refractory period tests must be the same because the same current, pulse duration, and anode are employed. We tried to ensure that this region was also stimulated by that cathode in the direction test by appropriately adjusting the number of pulses per train. According to the counter model of spatiotemporal integration in the substrate for the rewarding effects of MFB stimulation (Gallistel, 1975, 1978; Gallistel et al., 1981; Yeomans, 1975), the same region would be stimulated by the required current in the direction test if excitation occurred only at the cathode and the number of pulses per train in the direction test was set to the average of the required number of single pulses in the refractory period and collision tests. This should be true for all pulse durations, provided that each reward neuron fires once per pulse and that the strengthduration functions for all the stimulated reward neurons have the same chronaxie.

Collision tests on subjects D-6, D-29, and D-34 were carried out with two sets of currents. Consequently, two sets of direction tests were carried out on these subjects. One was conducted with the number of pulses set to the required number of single pulses from the collision test in which the higher currents were employed (high-current tests) and the other with the number of pulses set to the required number of pulses from the collision test in which the lower currents were employed (lowcurrent tests). Thus, eight strength-duration curves, four per cathode, were collected from subjects D-6, D-29, and D-34.

\section{Histology}

When all tests were completed, the animals were injected intraperitoneally with a lethal dose of sodium pentobarbital and perfused intracardially with physiological saline followed by $10 \%$ formalin. The heads were detached and placed in a stereotaxic instrument in order to remove the electrode assembly. This was done by carefully drilling through the bone surrounding the dental acrylic cap, which could then be easily removed without disturbing the electrode tips. The distance between the tips was measured with a micrometer.

The brains were removed from the skull and placed in $10 \%$ formalin for at least $24 \mathrm{hr}$. They were then frozen and sliced into $40 \mu \mathrm{m}$ sections that were later stained with thionin. The Pellegrino atlas was used as a reference in locating the electrode tips (Pellegrino et al., 1979).

\section{Results and Discussion}

\section{Histology}

The location of the electrode tips is shown in Figure 2, with the anterior placements on the left and the posterior placements on the right. The drawings are tracings of the atlas plates (Pellegrino et al., 1979) that best correspond to the sections containing the electrode tip tracks. All anterior and posterior placements were found to be in or on the borders of the LH and VTA, respectively. The VTA electrodes appear to be clustered just rostral to the intended target, while the anterior electrodes were scattered widely throughout the LH. A question mark is set beside the anterior placement of M-20 to denote our uncertainty about the exact location of that electrode tip.

\section{Strength-duration functions: high-current range}

The strength-duration curves obtained at the high currents are presented in Figure 3. The left-hand column consists of curves obtained with an LH cathode, while the central column consists of curves obtained with a VTA cathode. In both cases, the required-current values obtained with a depth cathode and a skull screw anode are represented as open triangles, while the values obtained with a depth cathode and a depth anode are represented by filled inverted triangles.

Visual inspection of these data reveals that the functions obtained with the LH cathode differ, while those obtained with a VTA cathode are similar. This contrast is heightened by comparing the ratios of each pair of curves. The curves representing the filled circles in the right-hand column were generated by dividing the values obtained with a given $\mathrm{LH}$ cathode and a VTA anode ( $\left.\mathrm{LH}^{-} \mathrm{VTA}^{+}\right)$by those obtained with the same cathode and a skull screw anode $\left(\mathrm{LH}^{-} \mathrm{SS}^{+}\right)$; the curves designated by the open circles represent the ratios of the values from the $\mathrm{VTA}^{-} \mathrm{SS}^{+}$and $\mathrm{VTA}^{-} \mathrm{LH}^{+}$conditions.

Note that the ratio curves derived from the results obtained with the VTA cathodes are nearly flat. This indicates that the strength-duration functions on which they are based are roughly parallel on logarithmic coordinates. Hence, it appears that as pulse duration was increased, the ratio of the rewarding effects of stimulating with the VTA- $\mathrm{LH}^{+}$and $\mathrm{VTA}^{-} \mathrm{SS}^{+}$configurations did not change. These results show no evidence of a behaviorally effective anodal block.

In contrast, all of the ratio curves representing the results obtained with the $\mathrm{LH}$ cathodes are ascending. That they are not flat shows that the underlying strength-duration functions have different shapes and that the ratio of the rewarding effects of stimulating the $\mathrm{LH}^{-} \mathrm{VTA}^{+}$and $\mathrm{LH}^{-} \mathrm{SS}^{+}$configurations varied with the pulse duration. That they are ascending shows that as pulse duration was increased, relatively more current was required in the $\mathrm{LH}^{-} \mathrm{VTA}^{+}$condition than in the $\mathrm{LH}^{-} \mathrm{SS}^{+}$condition. 


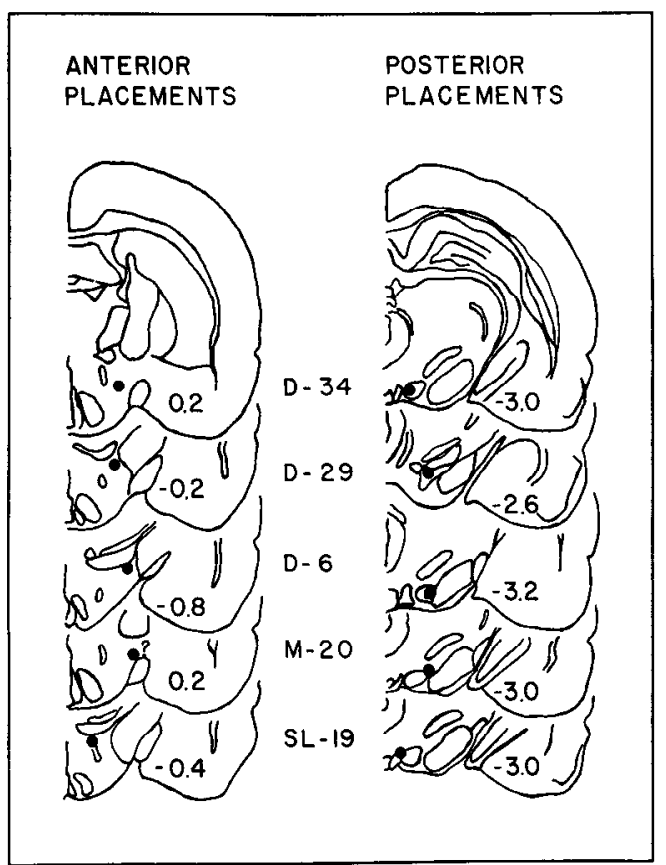

Figure 2. Tracings of pertinent sections from the Pellcgrino ct al. (1979) atlas. The filled circles indicate the location of the electrode tips. The numbers appearing at the top of each section refer to the distance (mm) of the section from bregma, and the alphanumeric located between each pair of sections refers to the identity of the subject.

This is consistent with the hypothesis that a behaviorally effective block was produced at the VTA site.

Statistical evaluation of the shapes of the strength-duration curves was carried out by means of nonlinear curve fitting. Matthews (1977) showed that behaviorally derived strengthduration curves obtained from MFB self-stimulation sites were fit fairly well by a rectangular hyperbola. We used the RS/1 software package (BBN Software Products) to fit the logarithms of the required currents with a hyperbolic function of the form:

$$
\log (I)=\log [R(1+C / D)]
$$

where $I$ is the required current; $R$, the rheobase or horizontal asymptote of the hyperbola; $C$, the chronaxie, the pulse duration at which the required current is twice the rheobase; and $D$, the pulse duration. Since the SE of the required currents at a given pulse duration was roughly proportional to the mean, fitting the hyperbola to the logarithms of the required currents minimized the heteroscedasticity of the residuals.

The solid curves in the left-hand and center panels of Figure 3 were fitted to the values obtained with a skull screw anode, whereas the dashed curves were fitted to the values obtained with depth anodes. The proportion of the variance accounted for by the fitted functions was high $(0.9984-0.9999)$.

On logarithmic coordinates, strength-duration curves that differ in rheobase are parallel but shifted vertically. Strength-duration curves that differ in chronaxie have different shapes: The larger the chronaxie, the steeper the decline of the function. A behaviorally effective anodal block should cause a strengthduration curve obtained with an appropriately positioned depth anode to decline more gradually than one obtained with the same cathode and a skull screw anode. Hence, the block will decrease the chronaxie of the fitted function. To test this hypothesis, we constructed ratios to evaluate the difference between the chronaxies of the curves obtained with a given cathode and either a depth or skull screw anode. The denominator of the $t$ ratio was the square root of the sum of the squared SE of the two chronaxie estimates.

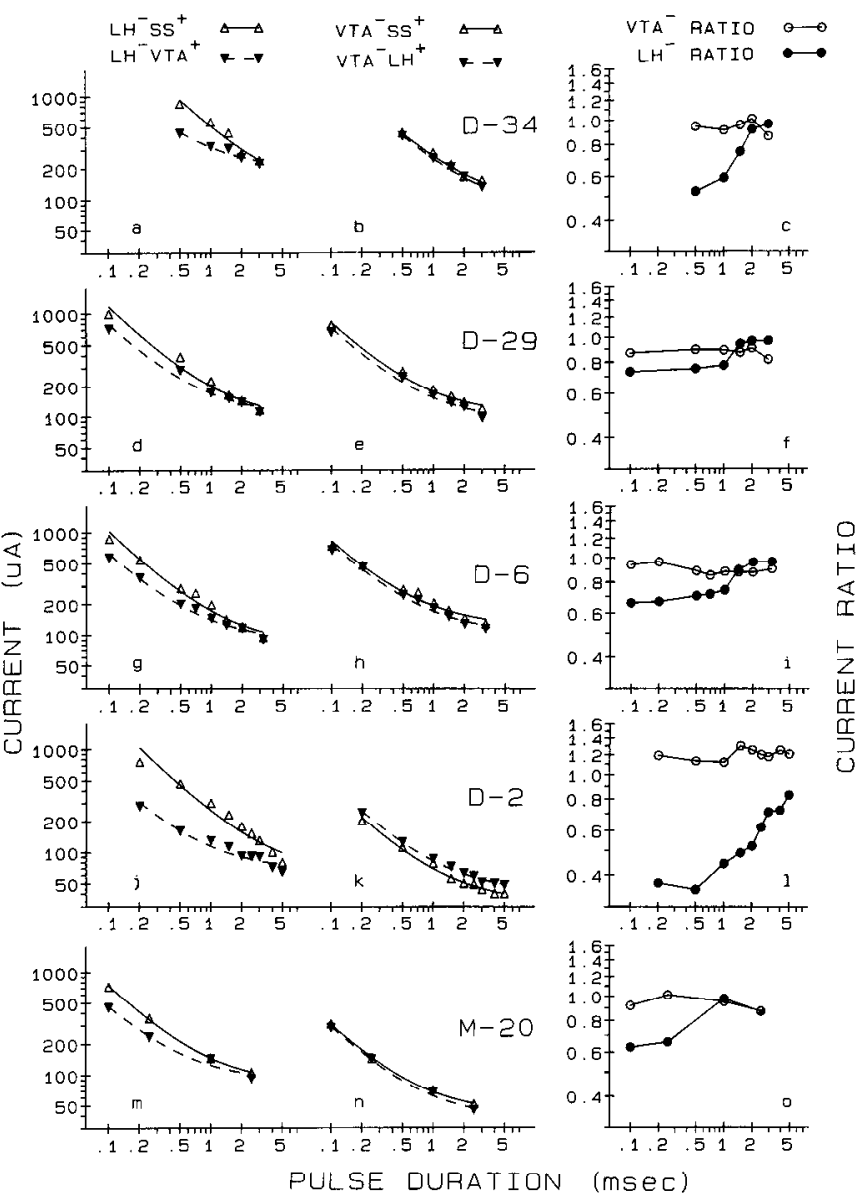

Figure 3. Results of the direction test, high-current data. Each pair of strength-duration curves in the left column was obtained with an $\mathrm{LH}$ cathode, while each pair of strength-duration curves in the center col$u m n$ was obtained with a VTA cathode. The open triangles joined by a solid line in the left and center columns represent data points obtained with a skull screw anode, and the filled inverted triangles joined by a dashed line represent points obtained with a depth anode. The curves in the right column are the ratios of each pair of strength-duration curves from the left and center columns. The filled circles denote the ratios of curves obtained with an LH cathode, and the open circles denote the ratios of curves obtained with a VTA cathode.

Table 1 (High current) lists the results of the statistical comparison of chronaxies. For each subject the chronaxie of the $\mathrm{LH}^{-} \mathrm{VTA}^{+}$curve was significantly shorter than the chronaxie of the $\mathrm{LH}^{-} \mathrm{SS}^{+}$curve. In contrast, the chronaxies of the VTA ${ }^{-} \mathrm{LH}^{+}$ and VTA-SS ${ }^{+}$curves were not significantly different. These results confirm and strengthen the visual impression conveyed by Figure 3; even the smallest of the shape differences between curves obtained with LH cathodes (panel $d$ ) was statistically reliable ( $p=0.0014$; one-tailed), whereas the largest of the shape differences between curves obtained with VTA cathodes was not $(p=0.2082$; one-tailed).

\section{Collision data}

\section{High currents}

The simplest anatomical arrangement consistent with the strength-duration data is one in which fibers stimulated by the LH cathode descend through the MFB at least to the level of the VTA, where an appropriately timed block can disrupt conduction. Such fibers should undergo collision when both of the depth electrodes serve as cathodes, the skull screws are used as the anode, and one of these sites is stimulated shortly after the 


\begin{tabular}{|c|c|c|c|c|c|c|}
\hline \multirow{2}{*}{$\begin{array}{l}\text { Sub- } \\
\text { ject }\end{array}$} & \multirow[b]{2}{*}{ Cathode } & \multicolumn{2}{|c|}{ Chronaxie } & \multirow[b]{2}{*}{$t$} & \multirow[b]{2}{*}{$d f$} & \multirow[b]{2}{*}{$p^{a}$} \\
\hline & & SS & Depth & & & \\
\hline D-34 & LH & 4065 & 686 & 2.12 & 36 & $0.021^{*}$ \\
\hline D-34 & VTA & 1943 & 2113 & -0.17 & 36 & 0.43 \\
\hline D-29 & LH & 1174 & 685 & 3.08 & 92 & $0.0014^{*}$ \\
\hline D-29 & VTA & 701 & 725 & -0.29 & 92 & 0.39 \\
\hline D-6 & LH & 1220 & 617 & 3.91 & 60 & $0.00050^{*}$ \\
\hline D-6 & VTA & 583 & 645 & -0.76 & 60 & 0.23 \\
\hline$D-2$ & LH & 3207 & 722 & 2.92 & 32 & $0.0032^{*}$ \\
\hline D-2 & VTA & 1140 & 1035 & 0.52 & 32 & 0.30 \\
\hline M-20 & LH & 823 & 433 & 4.72 & 11 & $0.00050^{*}$ \\
\hline M-20 & VTA & 644 & 711 & -0.85 & 9 & 0.21 \\
\hline
\end{tabular}

\begin{tabular}{|c|c|c|c|c|c|c|}
\hline \multirow{2}{*}{$\begin{array}{l}\text { Sub- } \\
\text { jcct }\end{array}$} & \multirow[b]{2}{*}{ Cathode } & \multicolumn{2}{|c|}{ Chronaxie } & \multirow[b]{2}{*}{$t$} & \multirow[b]{2}{*}{$d f$} & \multirow[b]{2}{*}{$p$} \\
\hline & & SS & Depth & & & \\
\hline D-34 & LH & 766 & 687 & 0.34 & 36 & 0.37 \\
\hline D-34 & VTA & 1221 & 1047 & 0.71 & 36 & 0.24 \\
\hline D-29 & LH & 868 & 868 & 0.00 & 8 & 0.50 \\
\hline D-29 & VTA & 823 & 851 & -0.14 & 8 & 0.45 \\
\hline D-6 & LH & 937 & 921 & 0.12 & 60 & 0.45 \\
\hline D-6 & VTA & 609 & 647 & -0.52 & 60 & 0.30 \\
\hline SL-19 & LH & 1594 & 1308 & 0.90 & 38 & 0.19 \\
\hline SL-19 & VTA & 1404 & 1325 & 0.34 & 38 & 0.37 \\
\hline
\end{tabular}

Data were obtained with a given depth electrode serving as the cathode and either the skull screws (SS) or the second depth electrode as the anode.

- Asterisk indicates a significant difference $(p \leq 0.05)$.

other. The central column of Figure 4 shows that this prediction was borne out. Note that at the longer C-T intervals the effectiveness of the stimulation is near 1.0 , indicating that the rewarding effects of LH stimulation summated almost perfectly with the rewarding effects of VTA stimulation. As the C-T interval is reduced, the effectiveness of the stimulation falls dramatically, an effect consistent with the collisions of orthodromic and antidromic action potentials in axons that coursc through both stimulation fields. This interpretation is strengthened by the similarity of the two sets of curves obtained when the $\mathrm{C}$ pulses were delivered to the $\mathrm{LH}$ and the $\mathrm{T}$ pulses to the VTA (filled circles) and when this order was reversed (open circles). Becausc of the bidircctional nature of axonal conduction, such a reversal should not alter the timing or likelihood of collision.

The C-T interval at which the step-like change in effectiveness occurs in Figure 4 is referred to as the "collision interval" and is interpreted as the sum of the interelectrode conduction time and the axonal refractory period (Bielajew and Shizgal, 1982; Shizgal et al., 1980a). In order to derive estimates of conduction velocity from the data in Figure 4, we obtained refractory period estimates using Yeomans' (1975) method. This procedure is essentially similar to the collision test, except that both $\mathrm{C}$ and $T$ pulses are applied to the same electrode.

The results obtained by this means are shown in the left-hand column of Figure 4 . The decline in the effectiveness values over the shortest $\mathrm{C}-\mathrm{T}$ intervals suggests local potential summation (Yeomans et al., 1979), whereas the later increase in effectiveness from near-zero values suggests that the most excitable reward neurons stimulated at these sites have absolute refractory periods as short as $0.4-0.6 \mathrm{msec}$ (Bielajew et al., 1982; Yeomans, 1975, 1979).

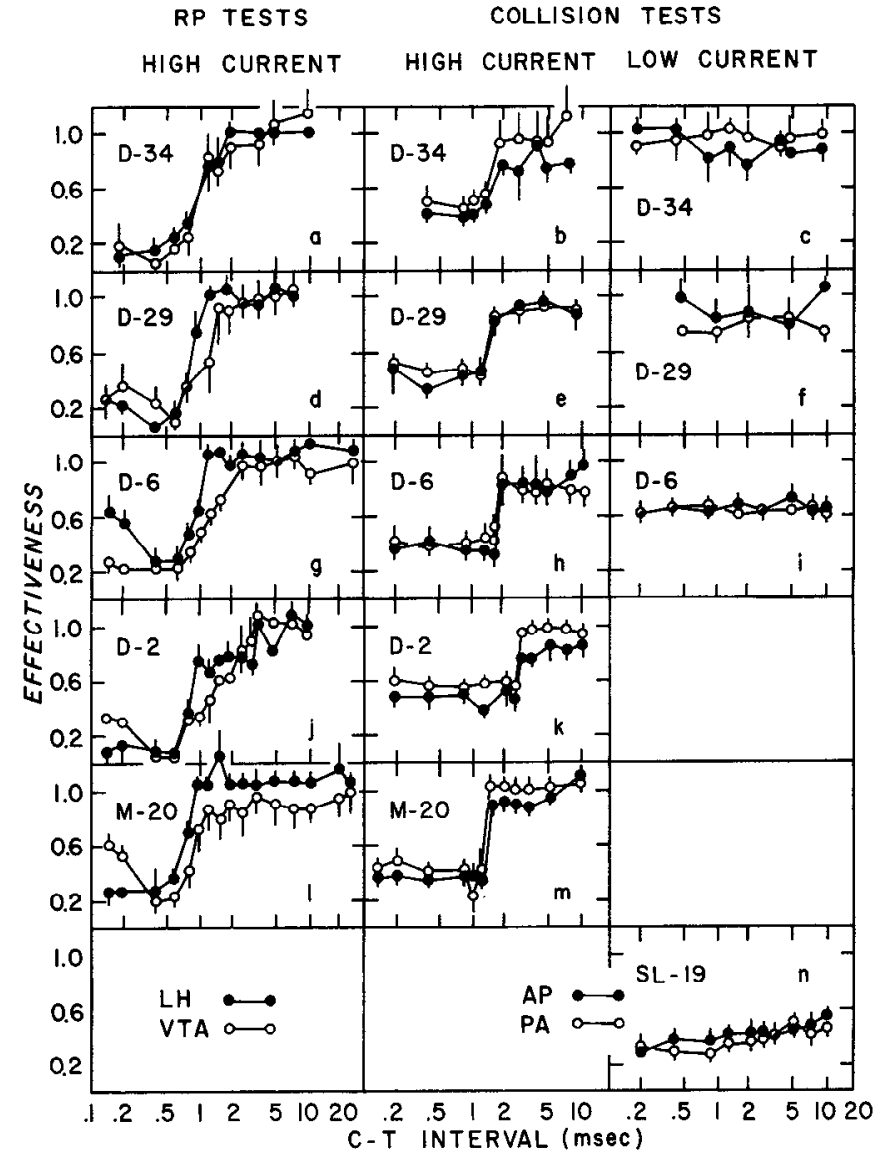

Figure 4. Results of the collision and refractory period tests for each subject. The effectiveness of paired-pulse stimulation is plotted as a function of $\mathrm{C}-\mathrm{T}$ interval. The refractory period curves obtained with currents at which collision effects were observed are shown in the left column, whereas the collision curves obtained with high and low currents are shown in the center and right columns, respectively. The filled circles represent LH refractory period data in the left column and AP collision data in the middle and right columns. The open circles represent VTA refractory period data and PA collision data. Note that the collision tests were not carried out at low currents with rats D-2 and M-20, and the refractory period tests were not carried out with rat SL-19. The collision data for rat SL-19 are listed under "low currents" because negative results were obtained for this subject in the pilot collision test.

We computed conduction velocity estimates by subtracting an estimate of the refractory period from an estimate of the collision interval and then dividing this difference into the interelectrode distance. The estimates computed from the data in Figure 4 ranged from 1.5 to $4.3 \mathrm{~m} / \mathrm{sec}$, values compatible with the results of previous behavioral studies of MFB reward neurons (Bielajew and Shizgal, 1982; Shizgal et al., 1980a). The exact means used to arrive at these estimates of conduction velocity have been described in detail elsewhere (Bielajew and Shizgal, 1982).

The rising portion of most of the collision curves in Figure 4 (central column) is quite steep, suggesting that the action potentials triggered by one electrode arrived in phase at the second stimulation site. In contrast, several of the ratio curves in Figure 3 tend to rise less abruptly, suggesting that in the direction test the arrival of action potentials at the hyperpolarized region was significantly staggered. We suspect that this was due to the wide range of utilization times. Because of the strength-duration relationship, neurons near the electrode tip will fire near the beginning of a stimulation pulse, while neurons near the edge of the effective stimulation field will fire near the end of the stim- 
ulation pulse. The contribution of this effect should have been much more pronounced in the direction test because the longest pulse durations tested were as much as 50 times longer than the $0.1 \mathrm{msec}$ pulses used in the collision test. A parallel phenomenon should affect the hyperpolarization block, further increasing the range of pulse durations over which the ratio curves rise.

\section{Low currents}

It is interesting to note that in Figure 4 the effectiveness values at the shortest $\mathrm{C}-\mathrm{T}$ intervals in the high-current collision test are greater than zero. This suggests that the electrodes were somewhat misaligned and that at least some of the fibers responsible for the rewarding effect passed through only one of the stimulation fields. This apparent misalignment provided the basis for another test of the hypothesis that anodal block was responsible for the difference in shape of the strength-duration curves obtained with the LH cathode.

As shown by the right-hand column of Figure 4, reducing the stimulation currents abolished the steplike change in effectiveness that we have attributed to collision. Presumably, the size of the effective fields was reduced to the point at which few, if any, reward fibers stimulated by one electrode were also stimulated by the other. It follows that a similar reduction in the size of the fields used in the direction test should have diminished or abolished the behaviorally effective anodal block.

\section{Strength-duration data: low-current range}

Figure 5 shows the strength-duration curves obtained using currents matched to those that failed to yield collision. These curves behave as predicted. In contrast to the difference in shape between the two sets of curves obtained with the LH cathode at the higher currents (Fig. 3, $a, d$, and $g$ ), parallel $\mathrm{LH}^{-}$curves were obtained at the lower currents from the same electrodes and subjects (Fig. 5, $a, d$, and $g$ ). As in the case of the higher currents, the VTA- curves are similar in shape. The same pattern is observed in the results from the subject (SL-19) that showed no evidence of collision at any of the currents tested. Consequently, all of the ratio curves (Fig. 5, $c, f, i$, and $L$ ) are flat, and in no case is the chronaxie of a curve obtained with a depth anode significantly different from the chronaxie of the curve obtained with the same cathode but with a skull screw anode (lower portion of Table 1). Again, the fitted functions accounted for a high proportion of the total variance (0.9986-0.9996).

The elimination of the shape difference by reducing the currents suggests that the region around the anode in which conduction was blocked was not significantly larger than the region around the cathode in which neurons were stimulated. Otherwise, a reduction in current that sufficed to eliminate the collision-like effect would not necessarily have sufficed to climinate the effect that we attribute to anodal block. This seems reasonable from a theoretical viewpoint. Given that the safety factor (Tasaki, 1953) must be considerably greater than unity to assure reliable conduction, the current sourced by an oncoming action potential must exceed the threshold current for excitation. If membrane resistance is fairly stable at subthreshold membrane potentials, this implies that to block conduction, the current entering from an anode must be larger than the exiting current required to initiate an impulse at the cathode. If so, the region in which a given current produced a hyperpolarization block at the anode is smaller than the region in which that current fires neurons at the cathode. This discrepancy would be sharpened if the anodal hyperpolarization reduced inactivation of sodium conductance that was present in the resting state (Hodgkin and Huxley, 1952). It follows from these arguments that it should be impossible to produce a behaviorally effective anodal block at currents comparable to those that fail to yield collision, but that with an appropriate alignment of electrodes, a collision effect may be obtained at currents comparable to those that fail
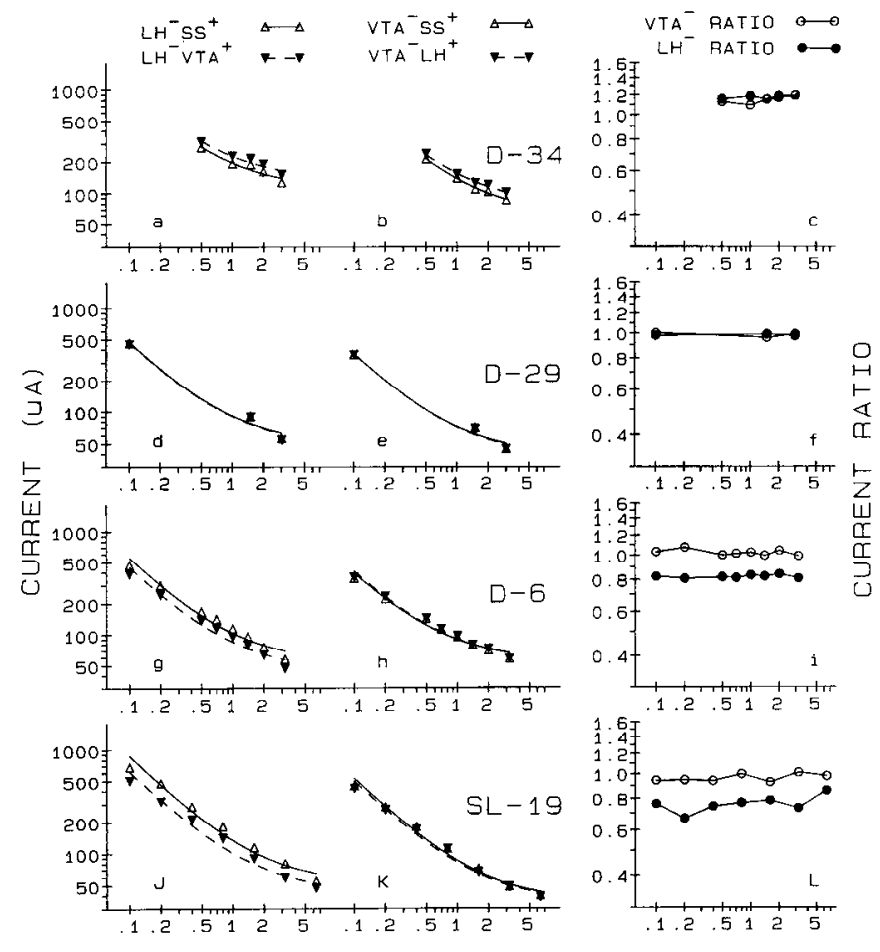

PULSE DURATION

(msec)

Figure 5. Results of the direction test, low-current data. The strengthduration functions and their ratios are plotted in the same manner as in Figure 3.

to yield a behavorially effective anodal block. The double dissociation achieved in the present study may be a consequence of the rather large difference (roughly twofold) in the two current ranges tested.

This argument and the partial nature of the collision block suggest that some of the fibers recruited by raising the current in the $\mathrm{LH}^{-} \mathrm{VTA}^{+}$condition should have escaped anodal block. If so, this explains how a decrease in the rewarding effect caused by the anodal block could have been offset by raising the current. In other words, this can account for the finding that self-stimulation was not eliminated at the longer pulse durations in the $\mathrm{LH}^{-} \mathrm{VTA}^{+}$condition and that the curves were merely bent upwards.

\section{Position of the strength-duration curves on the ordinate}

In our view, the refractory period and collision data are pertinent to two additional aspects of the direction results: The acrosssubject variance in the magnitude of the effect attributed to anodal block and the relative position on the ordinate of strengthduration curves obtained with an LH cathode and either a skull screw or a depth electrode. The following argument develops the idea that these aspects of the results can be understood in terms of across-subject variation in the relative densities of the reward substrate at the LH and the VTA.

Figure 3 shows that the convergence of the $\mathrm{LH}^{-}$strengthduration curves varies considerably across subjects and seems related to the relative position of the two sets of $\mathrm{SS}^{+}$curves (solid lines) on the ordinate. The effect is smallest in the subjects (D-6 and D-29) for which the curves lie at about the same level and largest in the subject (D-2) for which the curves obtained with the $\mathrm{LH}$ cathode lie in a higher current range than the curves obtained with the VTA cathode. To assess the significance of this relationship, we computed the correlation between two sets of quotients, one representing the relative position of the $\mathrm{LH}^{-} \mathrm{SS}^{+}$ and $\mathrm{VTA}^{-} \mathrm{LH}^{+}$curves on the ordinate and the other, the mag- 
nitude of the effect consistent with anodal block. We computed the first quotient by dividing the required current for the shortest pulse duration tested in the $\mathrm{LH}^{-} \mathrm{SS}^{+}$condition by the required current for that pulse duration tested in the VTA- $\mathrm{LH}^{+}$condition. The second quotient was obtained by dividing the highest point on the $\mathrm{LH}^{-}$ratio curve in Figure 3 by the lowest point. The correlation coefficient (Pearson's $r$ ) for the two sets of quotients was $0.916(p(3)=0.029)$.

The collision-like effect suggests that many of the reward fibers were stimulated by both electrodes, and the near-perfect summation observed in the collision test at the longer C-T intervals suggests that the signals elicited in these neurons, as well as in those stimulated by only one of the electrodes, converged onto the same final common path. Given that there appears to have been a large overlap in the efferent stages of the reward substrates activated by the two electrodes, the differences in the required currents in the $\mathrm{LH}^{-} \mathrm{SS}^{+}$and $\mathrm{VTA}^{-} \mathrm{LH}^{+}$conditions would appear to arise at the directly stimulated stage. One possibility is a difference in the excitability of the reward neurons at the $\mathrm{LH}$ and VTA levels of the MFB. However, the similarity of the refractory period curves obtained from the two sites is inconsistent with this notion [recall that the excitability of a fiber is highly correlated with its refratory period (Swadlow and Waxman, 1978)]. A much more appealing explanation is that in several of the subjects, the reward fibers were more tightly packed around the VTA electrode than around the LH electrode. If so, the number of reward neurons undergoing hyperpolarization block in these subjects should have been largest when the anode was in the VTA. We suspect that it is for this reason that the ratio of required currents in the $\mathrm{LH}^{-} \mathrm{SS}^{+}$and $\mathrm{VTA}^{-} \mathrm{SS}^{+}$conditions so accurately predicts the magnitude of the effect that we attribute to anodal block.

The putative difference in the density of reward fibers in the LH and VTA may also account for what otherwise might be a paradoxical aspect of the results obtained with the LH cathodes. If the efficiency of the stimulating current was independent of its angle and if excitation occurred only at the cathode, the two $\mathrm{LH}^{-}$curves should have overlapped at short durations and then diverged as pulse duration was increased to the point at which anodal hyperpolarization blocked conduction. Instead, these curves (Fig. 3, $a, d, g, j$, and $m$ ) converged as pulse duration was increased. This may have been due to a downward shift in the $\mathrm{LH}^{-} \mathrm{VTA}^{+}$curves caused by stimulation of fibers that passed near the VTA anode but not the LH cathode.

It is well known that fibers coursing near an anode may be stimulated, albeit inefficiently, wherever the current injected by the anode exists at suprathreshold density (Ranck, 1981). Thus, fibers that pass near the anode, but not the cathode, may be stimulatcd provided that the current is sufficiently intense. This effect should be confined to the conditions in which stimulation is delivered through two depth electrodes. The large surface area of the skull screws and their distance from the MFB preclude their injecting significant currents into neurons that pass near the inactive depth electrode but not near the depth cathode. Thus, when the skull screw is used as the anode, excitation should occur exclusively in the region of the cathode, whereas when two depth electrodes are used, the excited region around the cathode may be supplemented by a smaller region of excitation near the anode. If so, less current should be required to elicit self-stimulation when two depth electrodes are used than when current passes between the skull screws and one of the depth electrodes. Such an effect translates into a downward shift of the strength-duration curve obtained with two depth electrodes. If reward neurons were indeed more densely packed around the VTA electrode, then this mechanism should have contributed preferentially to the curves obtained using this electrode as the anode.

As predicted by this argument, there is a systematic relation- ship betwen the degree to which the $\mathrm{LH}^{-} \mathrm{VTA}^{+}$curves are so shifted and the relative positions of the corresponding $\mathrm{LH}^{-} \mathrm{SS}^{+}$ and $\mathrm{VTA}^{-} \mathrm{SS}^{+}$curves. We chose the value of the $\mathrm{LH}^{-}$ratio $\left(\mathrm{LH}^{-} \mathrm{SS}^{+} / \mathrm{LH}^{-} \mathrm{VTA}^{+}\right)$for the lowest pulse duration tested as an index of the shift in the strength-duration curves obtained in the $\mathrm{LH}^{-} \mathrm{VTA}{ }^{+}$condition. The lower the ratio, the larger the downward shift. These values are highly correlated with the index used above for the relative positions of the curves obtained with the skull screw anodes: the ratio of the required currents for the shortest pulse duration tested in the $\mathrm{LH}^{-} \mathrm{SS}^{+}$and $\mathrm{LH}^{-} \mathrm{VTA}{ }^{+}$conditions $(r=-0.904, p(3)=0.035)$.

The inefficiency of the proposed stimulation mechanism predicts that lower currents would activate relatively few neurons passing near the anode but not the cathode. Indeed, the shifts were much larger and more systematic when higher (Fig. 3), rather than lower (Fig. 5), currents were used. Since the ratio curves in Figure 5 are flat, we used the average of all the points on each curve as the index of the vertical shift in the low-current conditions. Whereas the lowest ratio obtained in the high-current $\mathrm{LH}^{-} \mathrm{VTA}^{+}$condition was 0.35 , the lowest value for the lowcurrent tests carried out with this configuration was 0.76 . In the low-current condition, the correlation between the shift in the strength-duration curves and the ratio of $\mathrm{LH}^{-} \mathrm{SS}^{+}$and VTA-SS ${ }^{+}$ currents for the shortest pulse duration used in the collision and refractory period tests was not significant $(r=-0.772, p(2)=$ 0.228 ).

Inconsistent with this hypothesis are the three cases where the curve obtained with the depth anode lies above the curve obtained with the skull screw anode (Figs. $3 k, 5, a$ and $b$ ). These results are also inconsistent with another mechanism that might have shifted the curves along the ordinate: the dependence of the threshold for axonal excitation on the angle of current flow (Ranck, 1981; Rushton, 1927). Given that the threshold is lowcst when the direction of current flow is parallel to the long axis of the fibers, this mechanism should cause the curves obtained with a given cathode and a depth anode to fall below those obtained with the same cathode and a skull screw anode. In addition, one would have expected the curves obtained with the VTA cathode to be shifted to the same degree as those obtained with the LH cathode. This was not the case. The failure of these predictions may reflect the fact that the stimulation field was a dipole with a short interpolar distance, a field in which the lines of flux are rarely parallel. In any case, the upward shifts are small and are seen in only 3 of the 18 sets of curves in Figures 3 and 5 .

\section{Anatomical implications of the direction results}

Figure $6 a$ represents the simplest anatomical arrangement consistent with the view that a behaviorally effective block was produced only with an LH cathode and a VTA anode. The somata of the neurons responsible for the difference in the shape of the $\mathrm{LH}^{-} \mathrm{VTA}^{+}$and $\mathrm{LH}^{-} \mathrm{SS}^{+}$strength-duration curves lie in the forebrain and project caudally. We regard this model as the most plausible and parsimonious explanation of the results. Nonetheless, more complex models can be constructed to account for these data. For example, Figure $6 b$ represents a neuron with a branching axon. The tips of both electrodes are located along the same branch, downstream from the branch point. The directly stimulated branch does not transmit reward signals, but the second branch does. In order to reach the terminals that transmit reward signals to the next stage, impulses must conduct antidromically along the stimulated branch. Although the behaviorally relevant direction of conduction is descending, the soma of this cell could lie caudal to the VTA electrode. Because of the more numerous and unusual assumptions it embodies, we regard this model as less attractive than the one in Figure $6 a$.

Even if the simpler model in Figure $6 a$ proves correct, a role 


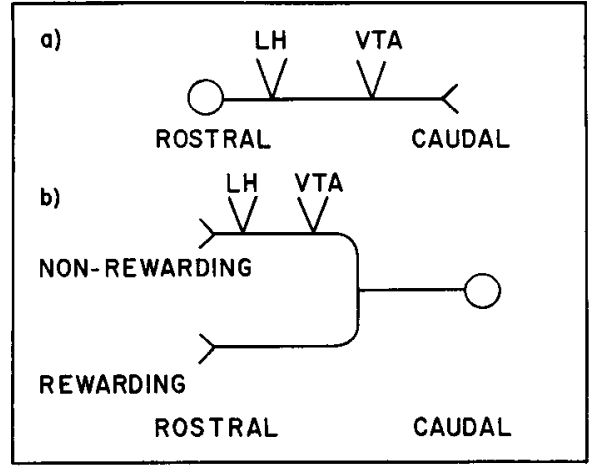

Figure 6. Examples of anatomical arrangements that could explain the direction results: $a$ represents the simplest case in which the somata of the directly stimulated reward neurons lie in the forebrain and project rostrocaudally, whereas in $b$ the electrodes are located downstream from a branch point and stimulate the branch that does not transmit reward signals (Non-rewarding). The branch that does contribute to the reward effect (Rewarding) is activated indirectly as a result of stimulating the other branch.

for ascending neurons in the rewarding effects of MFB stimulation cannot be ruled out. According to the arguments presented above, the results of the direction test pertain to the reward fibers responsible for the collision effect. They cannot shed light on whether ascending fibers were among those that escaped the collision block. It is also possible that a direction test carried out in other portions of the MFB would reveal some ascending reward fibers. Finally, the apparent difference in the density of reward fibers at the LH and VTA sites in some subjects introduced a bias toward the detection of descending neurons.

\section{Discussion}

\section{Significance of the technique: relation to other} techniques for inferring direction

Most previous studies aimed at inferring the direction in which MFB reward fibers project made use of methods other than anodal block for interfering with conduction between the stimulation site and later stages of the reward circuit. These methods include lesions (Olds and Olds, 1969; Stellar and Neeley, 1982) and the injection of local anesthetics (Nakajima, 1972; Stein, 1969). The principal problem with these methods is that they fail to distinguish between very different anatomical arrangements in which such disruptions may alter the transmission of reward signals. Consider the diagrams in Figure 7. In each case, stimulation is applied through the $S$ electrode, and conduction is disrupted at the site designated L. (For the purposes of these examples, except for $7 d$, knife cuts and injections of local anesthetic are logically equivalent to lesions.) Figure 7, $a-c$, depicts disconnection of the stimulation site from downstream elements responsible for the rewarding effect. That all three lesions will attenuate or eliminate self-stimulation demonstrates one of the ambiguities inherent in the lesion approach - one cannot know from the behavioral data whether the lesion damaged the directly stimulated cells, their immediate efferents, or cells located at yet later stages of the efferent pathway. Figure $7 d$ shows a special case where a lesion or a knife cut fails to directly interrupt the pathway between the stimulation site and the terminals of the directly stimulated cells. Nonetheless, the separation of the distal portion of the axon from the soma would likely lead to its degeneration and hence to behavioral consequences indistinguishable from those of the lesions in Figure $7, a-c$. This example is particularly telling because it demonstrates how, in principle, direction cannot be inferred from lesion data; the consequences of the lesions in Figures 7, $a$ and $d$, are the same
$S=S T I M U L A T I N G$ ELECTRODE

$L=$ LESIONING ELECTRODE

a)

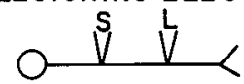

b)

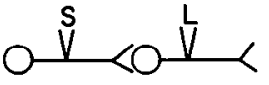

c)

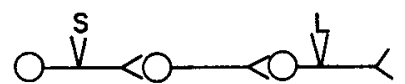

d)

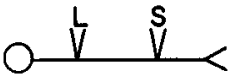

e)

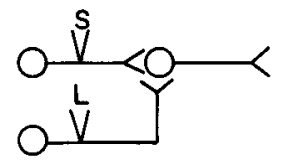

Figure 7. Hypothetical examples to illustrate the anatomical ambiguity inherent in the results of lesion experiments. Despite the very different anatomical linkages between the stimulating and lesioning sites, the lesion could produce the same behavioral effect in each case-a reduction in the effect of the stimulation.

despite the fact that in one case the lesion is upstream from the stimulation site, whereas in the other case it is downstream. Figure $7 e$ introduces yet another amibiguity: A lesion might disrupt self-stimulation without damaging either the directly stimulated cells or any of their efferents. Such an effect could arise if the lesion damaged a pathway that converged with efferents of the directly stimulated cells and gated transmission at the point of convergence.

In comparison to lesions and injection of local anesthetics, the technique introduced here offers several important advantages. First and foremost is the greatly increased likelihood that the blockade of conduction responsible for the behavioral effect is confined to the directly stimulated cells and occurs between the stimulation site and the terminals that transmit the reward signal to subscquent stages of the substrate. It is the combination of the collision and direction tests that provides the ground for localizing the block to the directly stimulated cells. In order for collision to occur, the same cells must be stimulated by both electrodes. Since the direction test is carried out with the same electrodes and stimulation fields of comparable size, it is highly likely that the reward neurons undergoing hyperpolarization block in the region of the anode are sampled from this population. A second advantage is that, unlike a lesion, the hyperpolarization block reduces transmission to the next stage of the reward circuit only if the anode lies between the cathode and the terminals. A third advantage of the technique employed here is that the block is under precise temporal control. At the moment that the rat approaches and presses the lever, transmission is normal; the block is turned on only for the few milliseconds required to reduce the effectiveness of a stimulation pulse. In contrast, the lesioned rat is altered during the entire test. It must organize its response in the face of potentially disruptive side effects such as sensory neglect (Marshall et al., 1971) or motor dysfunction.

German and Holloway (1973) proposed an alternative to the lesion approach based on the bilateral convergence of MFB signals. They assumed that a maximal effect would be produced when the signals arrived simultaneously at the point of convergence. Direction was inferred by stimulating the left and right MFB at different anteroposterior levels and noting the sign of the phase difference at which stimulation was maximally effective. They reported a small effect that, given their assumptions, suggested rostrocaudal conduction of MFB reward signals. Yeomans and Koopmans (1974) took issue with their statistical and 
measurement methods. Using more powerful measurement techniques, we (Shizgal et al., 1980a) studied the effectiveness of bilateral MFB stimulation as a function of the interval between stimulation of the two sides. We found no changes in effectiveness over a wide range of intervals and, hence, failed to support the principal assumption underlying German and Holloway's experiment.

Szabo and his coworkers (Szabo and Milner, 1972, 1973; Szabo et al., 1972) were the first to use anodal hyperpolarization block to infer the direction in which MFB reward fibers project. Unfortunately, key controls were omitted from their experiments. First, they could not rule out the possibility that differences in the density of reward fibers at the tips of the two depth electrodes accounted for the differences in threshold observed when the polarity of the current was reversed. It is for this reason that we compared the results obtained with a given cathode and a depth anode to those obtained with the same cathode and a skull screw anode. Second, they did not systematically manipulate the pulse duration. Third, they did not ascertain independently that the tips of the two depth electrodes were linked by the axons of reward neurons. Hence, we do not believe that their data yield an unambiguous interpretation.

\section{The promise of the technique for future work}

We believe that the direction and collision techniques together constitute a promising tool for identifying the neural systems responsible for interesting behavioral effects of brain stimulation such as analgesia, feeding, and reward. What is special about these methods is that they provide a linkage between structural, physiological, and behavioral data. Traditional anatomical methods can be used to trace the pathways that course past sites where stimulation produces interesting effects but provide little if any clue as to which of these pathways is responsible for the effect of interest. Traditional recording methods can identify cells driven by stimulation at such sites but again provide no means of determining whether these cells mediate the effect of interest or some other effect of the stimulation. Traditional stimulation techniques provide a rough localization of the sites at which interesting behavioral or physiological changes are produced but provide little information about the physiological and anatomical properties of the cells mediating these effects. In contrast, the collision and direction techniques combine these three types of information; they provide a basis for inferring the trajectory of the ncurons responsible for the effect of interest, the direction in which such neurons project, and the velocity at which action potentials propagate along their axons.

\section{Significance of the findings}

The findings of this study tell us something about what the MFB reward fibers are as well as something about what they are not. Our previous collision experiments (Bielajew and Shizgal, 1982; Shizgal et al., 1980a) and Yeomans' excitability studies (1975), 1979) suggest that the substrate for the rewarding effects of MFB stimulation consists, at least in part, of small myelinated neurons with conduction velocities ranging from roughly 1 to 8 $\mathrm{m} / \mathrm{sec}$ and refractory periods ranging from roughly 0.5 to 1.2 msec. The present experiment extends this characterization by adding information about the direction in which signals must travel along these fibers in order to produce their reward impact. In the most parsimonious anatomical arrangement compatible with our results, the somata of the neurons responsible for the collision effects lie in the forebrain and send descending axons through the MFB, at least to the level of the VTA. When coupled with experiments in which labeled 2-deoxyglucose is given to self-stimulating rats (Gallistel et al., 1985; Yadin et al., 1983; Yeomans et al., 1978), this information constrains the choice of nuclei most likely to include the somata. The likely candidates include regions of the septum, the vertical limb of the nucleus of the diagonal band of Broca, the bed nucleus of the stria terminalis, and the path neuron system of the MFB itself (Millhouse, 1969). The information from the present study is particularly important in arriving at this list of candidate structures because the 2-deoxyglucose method cannot distinguish rewardrelated cells from other stimulated neurons. In addition, it is not clear in existing 2-deoxyglucose data obtained from selfstimulating rats whether glucose utilization was increased in the somata of directly stimulated cells, in their terminals, or in postsynaptic elements. The list of candidate structures serves as a guide to electrophysiological studies (Matthews, 1978; Rolls, $1971 \mathrm{a}, \mathrm{b}$; Rompré and Shizgal, in press) in which one searches for the somata of reward neurons by comparing the characteristics of cells driven by stimulation at MFB reward sites to those inferred from psychophysical experiments (Gallistel et al., 1981). It is hoped that the identification of such cells will be the key to tracing the circuitry subserving self-stimulation of the MFB.

Our argument for rostrocaudal conduction of $\mathrm{MFB}$ reward signals and the results of our collision studies (Bielajew and Shizgal, 1982; Shizgal et al., 1980a) are incompatible with the view that the direct excitation of dopaminergic neurons is exclusively responsible for self-stimulation of the MFB: Dopaminergic neurons ascend through the MFB (Lindvall and Björklund, 1974) and have conduction velocities too low to account for the psychophysical data (Feltz and Albe-Fessard, 1972; German et al., 1980; Guyenet and Aghajanian, 1978; Maeda and Mogenson, 1980; Takigawa and Mogenson, 1977; Yim and Mogenson, 1980). Nonetheless, dopaminergic neurons may constitute a later stage in the reward circuit (Shizgal et al., 1980a; Wise, 1981) or, in a manner analogous to that depicted in Figure $7 e$, may play a permissive role by synaptically gating signals carried by reward neurons.

\section{References}

Bielajew, C., and P. Shizgal (1982) Behaviorally derived measures of conduction velocity for rewarding medial forebrain bundle stimulation. Brain Res. 237: 107-119.

Bielajew, C., M. Lapointe, I. Kiss, and P. Shizgal (1982) Absolute and relative refractory periods of the substrates for lateral hypothalamic and ventral midbrain self-stimulation. Physiol. Behav. 28: 125-132.

Deutsch, J. A. (1964) Behavioral measurement of the neural refractory period and its application to intracranial self-stimulation. J. Comp. Physiol. Psychol. 58: 1-9.

Feltz, P., and D. Albe-Fessard (1972) A study of an ascending nigrocaudate pathway. Electroencephalogr. Clin. Neurophysiol. 33: 179193.

Fibiger, H. (1978) Drugs and reinforcement mechanisms: A critical review of the catecholamine theory. Annu. Rev. Pharmacol. Toxicol. 18: $37-56$.

Gallistel, C. R. (1975) Spatial and temporal summation in the ncural circuit subserving brain stimulation reward. In Brain-Stimulation Reward, A. Wauquier and E. T. Rolls, eds., American Elsevier, New York.

Gallistel, C. R. (1978) Self-stimulation in the rat: Quantitative characteristics of the reward pathway. J. Comp. Physiol. Psychol. 92:977998.

Gallistel, C. R., P. Shizgal, and J. S. Yeomans (1981) A portrait of the substrate for self-stimulation. Psychol. Rev. 88: 228-273.

Gallistel, C. R., Y. Gomita, E. Yadin, and K. Campbell (1985) Forebrain origins and terminations of the medial forebrain bundle metabolically activated by rewarding stimulation or by reward-blocking doses of pimozide. J. Neurosci. 5: 1246-1261.

German, D., and F. Holloway (1973) Directionality of rewarding impulses within the medial forebrain bundle self-stimulation system of the rat. Science 179: 1345-1347.

German, D., M. Dalsass, and R. Kiser (1980) Electrophysiological examination of the ventral tegmental (A10) area in the rat. Brain Res. 181: 191-197.

Guyenet, P., and G. Aghajanian (1978) Antidromic identification of dopaminergic and other output neurons of the rat substantia nigra. Brain Res. 150: 69-84. 
Hodgkin, A. L., and A. F. Huxley (1952) A quantitative description of membrane current and its application to conduction and excitation in nerve. J. Physiol. (Lond.) 117: 500-544.

Hoebel, B. G. (1974) Brain reward and aversion systems in the control of feeding and sexual behavior. In Nebraska Symposium on Motivation, Vol. 22, J. K. Cole and T. B. Sonderegger, eds., University of Nebraska Press, Lincoln.

Kuffler, S., and E. Vaughan-Williams (1953) Small-nerve junctional potentials. The distribution of small motor nerves to frog skeletal muscle and the membrane characteristics of the fibres they innervate. J. Physiol (Lond.) 121: 289.

Lindvall, O., and A. Björklund (1974) The organization of the ascending catecholamine neuron systems in the rat brain as revealed by the glyoxcylic acid fluorescence method. Acta Physiol. Scand. (Suppl.) 412: 1-48.

Maeda, H., and G. Mogenson (1980) An electrophysiological study of inputs to neurons of the ventral tegmental area from the nucleus accumbens and medial preoptic-anterior hypothalamic areas. Brain Res. 197: 365-377.

Marshall, J., B. Turner, and P. Teitelbaum (1971) Sensory neglect produced by lateral hypothalamic damage. Science 174: 523-525.

Matthews, G. (1977) Neural substrate for brain stimulation reward in the rat: Cathodal and anodal strength-duration properties. J. Comp. Physiol. Psychol. 91: 858-874.

Matthews, G. (1978) Strength-duration properties of single units driven by electrical stimulation of the lateral hypothalamus in rats. Brain Res. Bull. 3: 171-174.

Millhouse, O. (1969) A Golgi study of the descending medial forebrain bundle. Brain Res. 15: 341-363.

Mundl, W. J. (1980) A constant-current stimulator. Physiol. Behav. 24: 991-993.

Nakajima, S. (1972) Effects of intracranial chemical injections upon self-stimulation in the rat. Physiol. Behav. 8: 741-746.

Nieuwenhuys, R., L. Geeraedts, and J. Veening (1982) The medial forebrain bundle of the rat. I. General introduction. J. Comp. Neurol. 206: 49-81.

Olds, M., and J. Olds (1969) Effects of lesions in medial forebrain bundle on self-stimulation behavior. Am. J. Physiol. 217: 1253-1264.

Pellegrino, L. J., A. S. Pellegrino, and A. J. Cushman (1979) A Stereotaxic Atlas of the Rat Brain, 2nd Ed., Plenum, New York.

Ranck, J. B., Jr. (1981) Extracellular stimulation. In Electrical Stimulation Research Techniques, M. M. Patterson and R. P. Kesner, eds., Academic, New York.

Rolls, E. (1971a) Involvement of brainstem units in medial forebrain bundle self-stimulation. Physiol. Behav. 7: 297-310.

Rolls, E. (1971b) Absolute refractory period of neurons involved in MFB self-stimulation. Physiol. Behav. 7: 311-315.

Rolls, E. T., M. J. Burton, and F. Mora (1980) Neurophysiological analysis of brain-stimulation reward in the monkey. Brain Res. 194: 339-357.

Rompré, P.-P., and P. Shizgal (in press) Electrophysiological characteristics of neurons in forebrain regions implicated in self-stimulation of the medial forebrain bundle in the rat. Brain Res.

Rushton, W. A. H. (1927) Effect upon the threshold for nervous excitation of the length of nerve exposed and the angle between current and nerve. J. Physiol. (Lond.) 63: 357-377.
Shizgal, P., C. Bielajew, D. Corbett, R. Skelton, and J. Yeomans (1980a) Behavioral methods for inferring anatomical linkage between rewarding brain stimulation sites. J. Comp. Physiol. Psychol. 94: 227237.

Shizgal, P., C. Bielajew, and I. Kiss (1980b) Anodal hyperpolarization block technique provides evidence for rostro-caudal conduction of reward related signals in the medial forebrain bundle. Soc. Neurosci. Abstr. 6: 422

Stein, L. (1969) Chemistry of purposive behavior. In Reinforcement and Behavior, J. Tapp, ed., Academic, New York.

Stellar, J., and S. Neeley (1982) Reward summation function measurements of lateral hypothalamic stimulation reward: Effects of anterior and posterior medial forebrain bundle lesions. In The Neural Basis of Feeding and Reward, B. Hoebel and D. Novin, eds., Haer Institute, Brunswick, ME.

Swadlow, H., and S. Waxman (1978) Activity-dependent variations in the conduction properties of central axons. In Physiology and Pathobiology of Axons, S. Waxman, ed., Raven, New York.

Szabo, I., and P. Milner (1972) Self-stimulation in rats: Tip alignment influences the effectiveness of bipolar electrodes. Brain Res. 48: 243250.

Szabo, I., and P. Milner (1973) Electrode tip alignment and self-stimulation: Influence of anodal hyperpolarization. Physiol. Behav. 11: 581-583.

Szabo, I., E. Nad, and C. Szabo (1972) Pole reversals and hypothalamic self-stimulation: Ascending spread of rewarding excitation. Physiol. Behav. 9: 147-150.

Takigawa, M., and G. Mogenson (1977) A study of inputs to antidromically identified neurons of the locus coeruleus. Brain Res. 135: 217230.

Tasaki, I. (1953) Nervous Transmission, Thomas, Springfield, IL.

Wise, R. A. (1978) Catecholamine theories of reward: A critical review. Brain Res. 152: 215-247.

Wise, R. A. (1980) Action of drugs on brain reward systems. Pharmacol. Biochem. Behav. (Suppl. 1) 13: 213-223.

Wise, R. A. (1981) Brain dopamine and reward. In Theory in Psychopharmacology, Vol. 1, S. J. Cooper, ed., Academic, New York.

Yadin, E., V. Guarini, and C. Gallistel (1983) Unilaterally activated systems in rats self-stimulating at sites in the medial forebrain bundle, medial prefrontal cortex, or locus coeruleus. Brain Res. 266: 39-50.

Yeomans, J. S. (1975) Quantitative measurement of neural post-stimulation excitability with behavioral methods. Physiol. Behav. 15: 593602.

Yeomans, J. S. (1979) The absolute refractory periods of self-stimulation neurons. Physiol. Behav. 22: $911-919$.

Yeomans, J., and H. Koopmans (1974) On the directionality of medial forebrain bundle fibers mediating self-stimulation. Science 183: 102 .

Yeomans, J. S., C. R. Gallistel, and M. Reivich (1978) Autoradiographic mapping of brain regions activated during self-stimulation using ${ }^{14} \mathrm{C}$-2-deoxyglucose. Soc. Neurosci. Abstr. 4: 230.

Yeomans, J. G., Matthews, R. Hawkins, K. Bellman, and H. Doppelt (1979) Characterization of self-stimulation neurons by their local potential summation properties. Physiol. Behav. 22: 921-929.

Yim, C., and G. Mogenson (1980) Electrophysiological studies of neurons in the ventral tegmental area of Tsai. Brain Res. 181: 301313. 